\title{
Detection of suspected brain infarctions on CT can be significantly improved with temporal subtraction images
}

\section{AUTHOR(S):}

Akasaka, Thai; Yakami, Masahiro; Nishio, Mizuho; Onoue, Koji; Aoyama, Gakuto; Nakagomi, Keita; lizuka, Yoshio; ... Satoh, Kiyohide; Yamamoto, Hiroyuki; Togashi, Kaori

\section{CITATION:}

Akasaka, Thai ...[et al]. Detection of suspected brain infarctions on CT can be significantly improved with temporal subtraction images. European radiology 2019, 29(2): 759-769

\section{ISSUE DATE:}

2019-02

URL:

http://hdl.handle.net/2433/235441

\section{RIGHT:}

This is a post-peer-review, pre-copyedit version of an article published in 'European radiology'. The final authenticated version is available online at: https://doi.org/10.1007/s00330-018-5655-0.; The full-text file will be made open to the public on 30 July 2019 in accordance with publisher's 'Terms and Conditions for Self-Archiving'; この論文は出版社版で ありません。引用の際には出版社版をご確認ご利用ください。; This is not the published version. Please cite only the published version. 
Title:

Detection of Suspected Brain Infarctions on CT Can Be Significantly Improved With Temporal Subtraction Images

\section{Authors:}

Thai Akasaka ${ }^{1}$, MD, Masahiro Yakami ${ }^{1,2}$, MD, PhD, Mizuho Nishio ${ }^{1,2}$, MD, PhD, Koji Onoue $^{1}$, MD, Gakuto Aoyama ${ }^{3}$, MS, Keita Nakagomi ${ }^{3}$, MS, Yoshio Iizuka ${ }^{3}$, MS, Takeshi Kubo $^{1}, \mathrm{MD}$, Yutaka Emoto ${ }^{4}, \mathrm{MD}, \mathrm{PhD}$, Kiyohide Satoh ${ }^{3}, \mathrm{PhD}$, Hiroyuki Yamamoto ${ }^{5}$, PhD, Kaori Togashi ${ }^{1}, \mathrm{MD}, \mathrm{PhD}$

1 Diagnostic Imaging and Nuclear Medicine, Kyoto University Graduate School of Medicine, 54 Kawahara-cho, Shogoin, Sakyo-ku, Kyoto, Kyoto 606-8507, Japan

2 Preemptive Medicine and Lifestyle-Related Disease Research Center, Kyoto University Hospital, 53 Kawahara-cho, Shogoin, Sakyo-ku, Kyoto, Kyoto 606-8507, Japan

3 Medical Imaging System Development Center, R\&D Headquarters, Canon Inc., 30-2, Shimomaruko 3-chome, Ohta-ku, Tokyo 146-8501, Japan.

4 Kyoto College of Medical Science, 1-3 Imakita, Koyamahigashi-cho, Sonobe-cho, Nantan, Kyoto 622-0041, Japan

5 Business Development Promotion Center, Corporate Planning Headquarters, Canon Inc., 30-2, Shimomaruko 3-chome, Ohta-ku, Tokyo 146-8501, Japan

\section{Corresponding author:}

Thai Akasaka

Phone: +81-80-3833-5559

FAX: +81-75-753-7631

Address: Diagnostic Imaging and Nuclear Medicine, Kyoto University Graduate School of Medicine,

54 Kawahara-cho, Shogoin, Sakyo-ku, Kyoto, Kyoto 606-8507, Japan

Email: thai.akasaka@gmail.com

takasaka@kuhp.kyoto-u.ac.jp 


\section{Title:}

Detection of Suspected Brain Infarctions on CT Can Be Significantly Improved With Temporal Subtraction Images 


\begin{abstract}
Objective:

To assess whether temporal subtraction (TS) images of brain CT improve the detection of suspected brain infarctions.
\end{abstract}

\title{
Methods:
}

Study protocols were approved by our institutional review board and informed consent was waived due to the retrospective nature of this study. Forty-two sets of brain CT images of 41 patients, each consisting of a pair of brain CT images scanned on two time points (previous and current) between January 2011 and November 2016, were collected for an observer performance study. The 42 sets consisted of 23 cases with a total of 77 newly developed brain infarcts or hyperdense artery signs confirmed by two radiologists who referred to additional clinical information, and 19 negative control cases. To create TS images, the previous images were registered to the current images by partly using a non-rigid registration algorithm, then subtracted. Fourteen radiologists independently interpreted the images to identify the lesions with and without TS images with an interval of over 4 weeks. A figure-of-merit (FOM) was calculated along with the jackknife alternative free-response receiver operating characteristic analysis. Sensitivity, number of false positives per case (FPC), and reading time were analyzed by the Wilcoxon signed-rank test.

\section{Results:}

The mean FOM increased from 0.528 to 0.737 with TS images $(\mathrm{P}<0.0001)$. The mean sensitivity and FPC improved from $26.5 \%$ and 0.243 to $56.0 \%$ and $0.153(\mathrm{P}<0.0001$ and $\mathrm{P}=0.239$ ), respectively. The mean reading time was 173 seconds without TS and 170 seconds with TS $(\mathrm{P}=0.925)$.

\section{Conclusion:}

The detectability of suspected brain infarctions was significantly improved with TS CT images. 


\section{Keywords:}

- multidetector computed tomography; stroke; brain infarction; computer assisted diagnosis; subtraction technique

\section{Key points:}

- Although it is established that MRI is superior to CT in the detection of strokes, the first choice of modality for suspected stroke patients is often CT.

- An observer performance study with 14 radiologists was performed to evaluate whether temporal subtraction images derived from a non-rigid transformation algorithm can significantly improve the detectability of newly developed brain infarcts on CT.

- Temporal subtraction images was shown to significantly improve the detectability of newly developed brain infarcts on CT.

\section{Abbreviations and acronyms:}

TS; temporal subtraction

FOM; figure of merit

FPC; false positives per case

DWI; diffusion weighted imaging

ADC; apparent diffusion coefficient

CAD; computer-aided detection

LDDMM; large deformation diffeomorphic metric mapping

FLAIR: fluid attenuation inversion recovery

HAS; hyperdense artery sign

EHL; early hyperacute lesion

CCL; cerebral cortex lesion

DWML; deep white matter lesion

AFROC; alternative free-response receiver operating characteristic

JAFROC; jackknife alternative free-response receiver operating characteristic

SNR; signal-to-noise ratio

CNR; contrast-to-noise ratio

GPU; graphics processing unit 


\section{Introduction}

The detection of fresh brain infarctions on CT is often a challenging task. Several reasons may be attributable. First, for the infarcted brain tissue to be conspicuous on CT, at least 6 hours after onset must elapse. Prior to this, the infarcted lesions may be depicted as subtly attenuated areas known as early CT signs, but considerable expertise is required for their detection [1-4]. Second, previous infarcts and chronic ischemic changes, which are likely present in patients with a high risk of ischemic events, often obscure fresh infarcts, especially in the deep white matter. Third, CT is prone to beam hardening artifacts which often hinder the detection of infarcts, particularly in the brain stem [5]. MRI suffers less from these disadvantages because DWI, and especially ADC maps, can elucidate fresh infarcts as rapidly as within an hour after onset in some cases [6]. Naturally, it is well established that MRI outperforms CT in the detection of strokes [7, 8]. Nevertheless, CT remains to be the first choice in many medical institutions due to its shorter acquisition time, better accessibility, fewer contraindications and lower cost [9].

The TS technique is one of the CAD techniques in which images are obtained by subtraction of two different sets of CT images to enhance the interval changes between them (hereafter, the two sets of CT are referred to as previous and current CTs). The two images must be registered before subtraction can be performed, and this can roughly be done in three ways - rigid, affine or non-rigid registration [10]. With rigid or affine transformation, subtle changes such as progression of brain atrophy or dilatation of the ventricles cause misregistrations, whereas non-rigid registration can cope with these subtle changes and generate higher quality subtraction images (Fig. 1).

TS has been shown to improve the detection of lung nodules on plain radiographs and thoracic CT, and TS with non-rigid registration similar to the method used in our study has been shown to improve detection of bone metastases on CT [11-14]. However, to the best of our knowledge, there is no report that has applied a TS technique to brain CT for the detection of newly developed brain infarcts. In this study we developed a TS technique for brain CT by use of a non-rigid image registration algorithm called LDDMM [15], and assessed whether TS images of brain CT can improve the detection of newly developed brain infarcts. 


\section{Materials and Methods}

\section{Subject Population}

Our clinical database was searched for radiology reports written between January 2011 and November 2016 with "infarct” as the keyword and "MR" as the modality. Next, for each candidate sequentially, pairs of brain CT images were searched that matched the following criteria: (i) scanned on two distinct time points, (ii) stored as thin-slice images ( $\leq$ $1 \mathrm{~mm}$ ), (iii) the presence of at least one low density area, regardless of size, in the brain parenchyma depicted only on the current CT, suggestive of a newly developed infarct between the two time points, and (iv) the absence of any focal lesions other than infarcts, such as tumor or hemorrhage. The exclusion criterion was TS image generation failure. The selected image pairs were retrospectively reviewed and their lesions were confirmed by consensus of two radiologists. The confirmation of an infarction was achieved by identifying high signal intensities in the suspected infarcted area on DWI $(B=1000)$ images and lower apparent diffusion coefficient values relative to the surrounding brain parenchyma when DWI images scanned within 5 days before or after the current CT were available, or by identifying isolated high signal intensities in the suspected infarcted area on FLAIR images scanned after the current CT were available. Additionally, all available clinical information including charts and future images was referred.

As control cases, our database was sequentially searched for pairs of brain CT images scanned between April 2013 and October 2016 that matched the first, second and fourth criteria for infarction-positive cases, but with no apparent newly developed infarcts.

\section{Subset Analysis}

To investigate whether our TS technique can enhance the detection of not only old infarcts that developed between the current and previous CTs but fresh infarcts as well, a subset of the whole data set was created whose positive cases consisted only of infarcts confirmed by DWI. The control group was kept the same. In addition, the following lesions were searched from the whole data set and their subset analyses were performed: HAS, defined as a high density area on the cerebral artery indicative of a thrombus; EHL, defined as an infarct depicted as a vaguely attenuated area on the current CT which was scanned less 
than 6 hours after onset, whose location matched the high signal intensity on DWI images, and suspected of causing the symptom; CCL, defined as an infarct which was mainly located on the cerebral cortex; DWML, defined as an infarct which was located in the deep white matter. All lesions were determined by consensus of two radiologists.

\section{Temporal Subtraction Technique}

TS images were generated in brief by the following steps: (i) registration of the previous CTs to the current CTs by rigid transformation followed by LDDMM and (ii) subtraction of the transformed previous CTs from current CTs. The details of the image processing are described in the online supplement material.

\section{Image Interpretation Session}

Fourteen readers consisting of 11 radiologists and 3 radiology residents with 3-25 years of clinical experience, independently interpreted two groups of thin-slice brain CT images: (A) 42 sets of current and previous CTs and (B) 42 sets of current, previous, and TS CTs. An in-house image viewer running on a Windows PC (Windows 10, Intel Core i7, 16 GB RAM) was used for the interpretation session. In order to reduce memory bias, randomly assigned seven and remaining seven readers were scheduled to interpret image groups $\mathrm{A}$ and $\mathrm{B}$ in their first session, then B and A in the second session, respectively, with an interval of more than four weeks between the two sessions. The order of the image sets was randomized for each reader. In order to control practice effects, readers were trained to the image viewer using six training cases prior to an actual observer study. The readers were blinded to all clinical data. The readers were asked to mark suspicious newly developed brain infarcts and intracranial thrombi with a likelihood level of diagnosis (1 to 100\%). No limits were set to the size of the lesion to mark or to the time allowed for interpretation of the images. If a single lesion was considered marked several times, the mark with the highest likelihood level was treated as the response. After interpretation of each case, the readers were asked to rate the confidence level of their interpretation (Survey 1: 1, very low; 2, low; 3, moderate; 4, high; 5, very high) and the usefulness of TS images (Survey 2: 1, useless; 2, not very useful; 3, somewhat useful; 4, very useful; 5, extremely useful) for each case. Reading time for each case was recorded. 


\section{Statistical Analysis}

The lesion-based sensitivity, case-based sensitivity and specificity, number of FPC, reading time and confidence level were compared between the two sessions using the Wilcoxon signed-rank test. For lesion-based analysis, a lesion with 51\% or higher likelihood of being an infarct was considered positive. For case-based analysis, cases with at least one positive lesion were considered positive. To evaluate observer performance, a FOM, equivalent to the area under the AFROC curve, was calculated along with the JAFROC analysis. Simply put, the AFROC curve is a lesion-based version of the ROC, which is a case-based analysis $[16,17]$. The analysis was conducted using the freely available JAFROC software (JAFROC, version 4; http://www.devchakraborty.com) with a random-readers and random-cases model [17]. Changes in the sensitivity for HAS, EHL, CCL, and DWML between with and without TS images were analyzed with the Wilcoxon signed-rank test.

\section{Results}

Twenty nine pairs of brain CT images of 28 patients matched the inclusion criteria by the search through our image database. No pairs matched the exclusion criteria, and 6 pairs were randomly chosen as training cases. The retrospective review of the remaining 23 pairs by the two radiologists revealed a total of 77 lesions. The 77 lesions comprised 74 brain suspected infarcts in 23 cases and 3 HAS in 3 cases. The 74 infarcts included 5 EHLs, 23 CCLs, and 9 DWMLs. The 3 HAS comprised two hyperdense middle cerebral artery signs and one hyperdense anterior cerebral artery sign. Of the 74 infarcts, 52 (70.3\%) infarcts in 22 cases, including 5 EHLs in 5 cases, were confirmed by DWI to be infarcts that developed between the previous and current CTs. Of the remaining 22 infarcts, 13 (17.6\%) infarcts in 5 cases were confirmed by FLAIR, 7 (9.5\%) infarcts in one case were confirmed by future CT, and the remaining 2 (2.7\%) infarcts in 2 cases were determined by previous and current CTs only. The mean size of the identified brain infarcts was $15.1 \mathrm{~mm} \pm 18.0$ and the number of infarcts per infarction-positive case was 3.2. A detailed list of all positive cases is shown in Table 1 and the statistics of the cases are summarized in Table 2. 
The processing time for image registration with LDDMM for each case ranged from 664.2 to 2343 seconds while the mean was 1189 seconds. There were no apparent registration failures.

The FOM, lesion-based sensitivity, case-based sensitivity and specificity, FPC, reading time, and results of the two surveys for the 14 radiologists are shown in Table 3 . The mean AFROC plot of all readers is shown in Fig. 2. The FOM improved for all radiologists. The mean FOM significantly improved from 0.528 to $0.737(\mathrm{P}<0.0001)$. The mean sensitivity on a lesion-based analysis significantly increased from $26.5 \%$ (286/1078) to $56.0 \%$ (604/1078) $(\mathrm{P}<0.0001)$ with the addition of TS images. The mean FPC decreased from 0.243 to $0.153(\mathrm{P}=0.239)$. The mean sensitivity on a case-based analysis significantly increased from 52.8\% (170/322) to 78.0\% (251/322) $(\mathrm{P}<0.0001)$ and the mean specificity on case-based analysis increased from $83.5 \%(222 / 266)$ to $90.6 \%(241 / 266)(\mathrm{P}=0.346)$.

The mean reading time was 173 seconds without TS and 170 seconds with TS $(\mathrm{P}=0.925)$. The radiologists' confidence in their interpretation (Survey 1) improved with TS, but not significantly ( $\mathrm{P}=0.733)$. The median of the confidence level was 3 for both with and without TS. The median of the usefulness of TS (Survey 2) was 4, and the minimum was 3.

The subset, whose positive cases consisted only of infarcts confirmed by DWI, comprised 14 positive cases with 42 infarcts (mean size, $14.1 \pm 10.0 \mathrm{~mm}$; number of lesions per infarction-positive case, 3.0) and 19 control cases. The FOM improved for all readers and its mean significantly improved from 0.521 to $0.750(\mathrm{P}<0.0001)$. The mean sensitivity on a lesion-based analysis significantly increased from 26.0\% (153/588) to 58.5\% (344/588) $(\mathrm{P}<0.0001)$ with the addition of TS images. The mean FPC decreased from 0.219 to 0.141 $(\mathrm{P}=0.628)$. The mean sensitivity on a case-based analysis significantly increased from $50.0 \%$ (98/196) to $80.1 \%(157 / 196)(\mathrm{P}=0.047)$ (Table 3).

The mean lesion-based sensitivities for HAS ( $\mathrm{N}=3$ ) slightly decreased from $31.0 \%$ $(13 / 42)$ to $28.5 \%(12 / 42)(\mathrm{P}=0.629)$, that for EHL $(\mathrm{N}=5)$ increased from $34.3 \%(24 / 70)$ to $67.1 \%(47 / 70)(\mathrm{P}<0.001)$, that for CCL $(\mathrm{N}=23)$ increased from $25.2 \%(81 / 322)$ to $57.1 \%$ $(184 / 322)(\mathrm{P}<0.0001)$, and that for DWML $(\mathrm{N}=9)$ increased from $19.8 \%(25 / 126)$ to $53.2 \%$ $(67 / 126)(\mathrm{P}<0.001)$.

Three representative cases in which the sensitivity improved drastically with the addition of TS CT images are shown in Figs 3 to 5. The first infarct is a CCL in the right 
frontal lobe, which was correctly marked by $21 \%$ (3/14) of the readers without TS and 100\% (14/14) with TS (Fig 3). The second infarct is a CCL in the left frontal lobe, which was correctly marked by none of the readers without TS and 93\% (13/14) with TS (Fig 4). The third infarct is an EHL in the left lateral lobe, which was correctly marked by $7.1 \%$ (1/14) of the readers without TS and 93\% (13/14) with TS (Fig 5).

On the contrary, there were a total of 3 cases that demonstrated detrimental effects of TS (i.e. showed a decrease in sensitivity). Two of the cases were a HAS and an EHL in the left cerebral hemisphere. They were both easily noticeable on the current CT and the decrease in sensitivity was not significant. The other case depicted a fresh infarct in the pons, and 21\% (3/14) of the readers were able to mark the lesion correctly without TS but none were able to with TS (Fig 6).

\section{Discussion}

Although the superiority of MRI to CT in the detection of strokes is well established, CT often remains to be the first choice for suspected stroke patients in clinical practice[7-9]. The main reason for this is likely due to the advantages of CT such as better accessibility, quicker acquisition time, fewer contraindications and lower cost [9]. Considering the advantages and prevalence of CT scanners, we believe that our result of improved detection of brain infarcts with TS CT images is significant. It is also noteworthy that we recruited as many as 14 readers for the observation performance study, which to our knowledge is unprecedented for this type of study.

The FOM among readers without TS varied from 0.21 to 0.67 and the lesion-based sensitivity varied from 0.12 to 0.40 . This large difference probably reflects the fact that the ability to detect infarcts varies greatly among the readers. However, the result that the FOM and sensitivity markedly improved for every reader shows that TS images can enhance this ability regardless of the reader's original detectability of brain infarcts (Table 3).

The mean reading time did not increase with the addition of TS CT images. Along with the improved detectability of brain infarcts and better confidence level and usefulness from the survey, the result suggests that the addition of TS CT images does not pose a burden on the reader as a whole. The question remains as to whether the decrease in time is a result of the readers' reduced viewing time of the current CTs, and as mentioned earlier, the case 
with a fresh infarct of the pons which revealed a detrimental effect of TS suggests the possibility that this may indeed be true.

The marked improvements in the detectability with TS observed in two CCLs (Fig 3 , 4) were probably due to the rather peculiar shape and locations of these lesions; they were both elongated and located roughly parallel to the brain sulci, rendering them indistinguishable between infarct and sulci. The TS images were successful in depicting these inconspicuous lesions quite apparently.

As mentioned earlier, CT is prone to beam hardening artifacts and they are prominent in the brain stem due to the surrounding skull base [5]. In the case where a detrimental effect was seen in the sensitivity of a fresh pons infarct (Fig 6), although the lesion is faint, it is detectable on the current CT. However, it is very inconspicuous on the TS image since it has been influenced by the different effects of beam hardening and noise on the current and previous CTs. This is possibly a disadvantage of the current version of the TS technique and needs improvement.

There were several limitations to this study. First, not all infarcts in the image set were confirmed by DWI. We addressed this problem by creating a subset whose positive cases consisted only of infarcts confirmed by DWI. The analysis of this subset revealed significant improvements in the mean FOM, lesion-based and case-based sensitivity and specificity, almost identical to that of the full image set.

Second, although we carefully defined the gold standard, we cannot completely rule out the possibility that the gold standard included other lesions such as demyelinating plaque or encephalitis.

Third, the interpretation session was carried out with thin-slice CT images only. In our interpretation session, the slice thickness of TS CT images was equal to that of the current image ( $\leq 1 \mathrm{~mm}$ ), but in a typical clinical setting, brain CT images reconstructed with a thickness of 4 to $5 \mathrm{~mm}$ are used as well for interpretation. Although thick-slice CT images ( $\geq 4 \mathrm{~mm}$ ) might aid the detection of early CT signs where the contrast between cortex and white matter is vague, it will likely be challenging to detect small infarcts that are less than 5 mm in size. Moreover, a guideline by the American College of Radiology for practice parameters of brain CT states that for brain CT the slice thickness should be no greater than 5 $\mathrm{mm}$ and as thin as possible for imaging of the cranial base [18]. A recent study showed that 
thin-slice $(0.625 \mathrm{~mm})$ brain CT yields better detectability of intraluminal thrombus than thick-slice (5 mm) CT [19]. As such, we did not consider this limitation to be critical in our study design. However, it is known that the SNR and CNR increases as the slice thickness of CT images increases [20], so there is a possibility that the detectability of EHLs without TS might have been underestimated. Therefore it would be premature to conclude that TS CT images significantly improve the detectability of EHLs when thick-slice CT images are used. Future work is necessary to investigate this.

Fourth, in order to create TS images with our technique, a previous brain CT must be available as thin-slice CT images. Although currently this may not often be the case, we expect that more CT images will be stored as thin-slice CT and the TS scheme can be more widely applied as the storage cost of images decreases with time and the utility of TS images is recognized.

Lastly, the mean processing time for image registration with LDDMM was approximately 20 minutes, which is not trivial considering the importance of detecting EHLs in the short time frame allowed. This will hopefully not be a significant problem since the computation time can be expected to reduce drastically when the algorithm is further optimized and the use of GPU is incorporated.

In summary, temporal subtraction CT images significantly improved the detectability of newly developed brain infarcts without compromising interpretation time and showed promise for helping clinicians in their detection.

\section{Acknowledgements}

The author K.T. has received funding from Canon Inc. The authors G.A., K.N., Y.I., K.S. and H.Y. are employees of Canon Inc. 


\section{References}

1. Tomura N, Uemura K, Inugami A, et al (1988) Early CT finding in cerebral infarction: obscuration of the lentiform nucleus. Radiology 168:463-7 . doi: 10.1148/radiology.168.2.3393665

2. Truwit CL, Barkovich AJ, Gean-Marton A, et al (1990) Loss of the insular ribbon: another early CT sign of acute middle cerebral artery infarction. Radiology 176:801806 . doi: 10.1148/radiology.176.3.2389039

3. Moulin T, Cattin F, Crépin-Leblond T, et al (1996) Early CT signs in acute middle cerebral artery infarction: Predictive value for subsequent infarct locations and outcome. Neurology 47:366-375 . doi: 10.1212/WNL.47.2.366

4. Tomsick TA, Brott TG, Chambers AA, et al (1990) Hyperdense middle cerebral artery sign on CT: Efficacy in detecting middle cerebral artery thrombosis. Am J Neuroradiol $11: 473-477$

5. Barrett JF, Keat N (2004) Artifacts in CT: Recognition and Avoidance. RadioGraphics 24:1679-1691 . doi: 10.1148/rg.246045065

6. Allen LM, Hasso AN, Handwerker J, Farid H (2012) Sequence-specific MR Imaging Findings That Are Useful in Dating Ischemic Stroke. RadioGraphics 32:1285-1297 . doi: 10.1148/rg.325115760

7. Chalela J a, Kidwell CS, Nentwich LM, et al (2007) Magnetic resonance imaging and computed tomography in emergency assessment of patients with suspected acute stroke: a prospective comparison. Lancet (London, England) 369:293-8 . doi: 10.1016/S0140-6736(07)60151-2

8. Fiebach JB, Schellinger PD, Jansen O, et al (2002) CT and diffusion-weighted MR imaging in randomized order: Diffusion-weighted imaging results in higher accuracy and lower interrater variability in the diagnosis of hyperacute ischemic stroke. Stroke 33:2206-2210 . doi: 10.1161/01.STR.0000026864.20339.CB

9. Birenbaum D, Bancroft LW, Felsberg GJ (2011) Imaging in acute stroke. West J Emerg Med 12:67-76 . doi: 10.1161/STR.0000000000000098

10. Crum WR, Hartkens T, Hill DLG (2004) Non-rigid image registration: theory and practice. Br J Radiol 77:S140-S153 . doi: 10.1259/bjr/25329214

11. Aoki T, Oda N, Yamashita Y, et al (2011) Usefulness of Computerized Method for 
Lung Nodule Detection in Digital Chest Radiographs Using Temporal Subtraction Images. Acad Radiol 18:1000-1005 . doi: 10.1016/j.acra.2011.04.008

12. Uozumi T, Nakamura K, Watanabe H, et al (2001) ROC analysis of detection of metastatic pulmonary nodules on digital chest radiographs with temporal subtraction. Acad Radiol 8:871-878 . doi: 10.1016/S1076-6332(03)80766-3

13. Aoki T, Murakami S, Kim H, et al (2014) Temporal Subtraction Method for Lung Nodule Detection on Successive Thoracic CT Soft-Copy Images. Radiology 271:255261 . doi: 10.1148/radiol.13130460

14. Sakamoto R, Yakami M, Fujimoto K, et al (2017) Temporal Subtraction of Serial CT Images with Large Deformation Diffeomorphic Metric Mapping in the Identification of Bone Metastases. Radiology 285:629-639 . doi: 10.1148/radiol.2017161942

15. Beg MF, Miller MI, Trouvé A, Younes L (2005) Computing large deformation metric mappings via geodesic flows of diffeomorphisms. Int J Comput Vis 61:139-157 . doi: 10.1023/B:VISI.0000043755.93987.aa

16. Swets JA (1979) ROC analysis applied to the evaluation of medical imaging techniques. Invest Radiol 14:109-21 . doi: 10.1097/00004424-197903000-00002

17. Chakraborty DP (2013) A Brief History of Free-Response Receiver Operating Characteristic Paradigm Data Analysis. Acad Radiol 20:915-919 . doi: 10.1016/j.acra.2013.03.001

18. John E. Jordan, Merita A. Bania, Kristine A. Blckham, Robert J. Feiwell, Steven W. Hetts EGH (2014) Acr - Asnr Practice Parameter for the Performance of Computed Tomography ( Ct ) of the Brain. Am Coll Radiol 1076:1-9

19. Inoue T, Nakaura T, Yoshida M, et al (2017) Diagnosis of small posterior fossa stroke on brain CT: effect of iterative reconstruction designed for brain CT on detection performance. Eur Radiol 27:3710-3715 . doi: 10.1007/s00330-017-4773-4

20. Tanaka C, Ueguchi T, Shimosegawa E, et al (2006) Effect of CT acquisition parameters in the detection of subtle hypoattenuation in acute cerebral infarction: A phantom study. Am J Neuroradiol 27:40-45 


\section{Figure and table legends}

Table 1. List of all infarction-positive cases and lesions used for the observer performance study. From the left are the age, sex, symptoms, presumed elapsed time since the last onset at the time of the current CT scan, number of lesions for all 23 infarction-positive cases, and the size, location, method of confirmation (DWI/FLAIR), classification

(HAS/EHL/CCL/DWML) of all 77 lesions. As the method of confirmation, a circle (०)

denotes that the lesion was confirmed by the image (DWI or FLAIR), a cross $(\times)$ denotes that the lesion could not be confirmed on the image, and a negative sign (-) denotes that the image did not exist. Note that patient number 3 appears twice since two cases were obtained at two different time points from this patient.

Table 2. Statistics of all cases used for the observer performance study. From the left are the number of cases, their age distribution according to their sex, and the scan intervals between the previous and current CT images for each group (all positive, DWI-positive only, and control cases). The table includes two positive cases obtained from one female patient. They were treated as independent patients for the calculation of the corresponding values.

Table 3. Results of the observer performance study. From the left are the FOM values, sensitivities per lesion, sensitivities per case, specificities per case, number of false positives per case, reading times and survey results of the 14 readers for all cases $(\mathrm{N}=42)$, and FOM values and sensitivities per lesion for the subset $(\mathrm{N}=33)$. The subset comprises positive cases consisting of DWI-positive infarcts only $(\mathrm{N}=14)$ and control cases $(\mathrm{N}=19)$. All values are mean except for the two surveys which are medians. An asterisk after the number indicates a significant improvement between without TS and with TS $(\mathrm{P}<0.0001)$.

Fig 1. Comparison of two temporal image subtraction techniques; rigid and non-rigid transformations. The (A) previous CT, (B) current CT, (C) TS CT using rigid registration only, and (D) TS CT using rigid registration refined with non-rigid registration (in this case, LDDMM) are shown. Rigid transformation and subtraction causes minor misregistrations of 
the brain sulci whereas LDDMM and subtraction can cope with these subtle changes and generate better subtraction images.

Fig 2. The mean AFROC plot of all readers. The vertical axis is the lesion localization fraction (equivalent to sensitivity) and the horizontal axis is the false positive fraction (equivalent to number of false positive cases divided by the total number of control cases). The detectability of brain infarctions with TS images is significantly better than that without TS.

Fig 3. A case of a 72-year-old male with a fresh infarct in the right frontal lobe (arrow). From the left the (A) previous CT, (B) current CT, (C) TS CT, and (D) DWI image (B=1000) are shown. The lesion was correctly marked by $21 \%$ (3/14) of the readers without TS and 100\% (14/14) with TS. Another fresh infarct is present in the right frontal lobe.

Fig 4. A case of an 82-year-old male with a fresh infarct in the left frontal lobe (arrow). From the left the (A) previous CT, (B) current CT, (C) TS CT, and (D) DWI image (B=1000) are shown. The lesion was correctly marked by none of the readers without TS and 93\% (13/14) with TS.

Fig 5. A case of a 78-year-old female with a fresh infarct in the left lateral lobe (dotted circle). From the left the (A) previous CT, (B) current CT, (C) TS CT, and (D) DWI image (B=1000) are shown. The lesion was correctly marked by $7.1 \%$ (1/14) of the readers without TS and 93\% (13/14) with TS.

Fig 6. A case of an 80-year-old female with a fresh infarct in the pons (arrow). From the left the (A) previous CT, (B) current CT, (C) TS CT, and (D) DWI image (B=1000) are shown. The lesion was correctly marked by $21 \%$ (3/14) of the readers without TS and none with TS. Note how the lesion is inconspicuous on the TS CT although they are apparent on the current CT and DWI images. An old right cerebellar infarct coexists in the current CT. 
22.9 Rt. cerebellar hemisphere

$5 \quad 14.4$ Rt. cerebellar hemisphere 5.6 Rt. cerebellar hemisphere 17.9 Lt. occipital cortex

$292 \quad F \quad$ Right hemiparalysis $47-48$

$3 \quad 72 \quad M \quad$ Dysbasia, left hemiparesis

$3 \quad 72 \quad M \quad$ Dysbasia, left hemiparesis 120

Unknown

$12.1 \mathrm{Lt}$. frontal cortex

22.8 Lt. cerebellar hemisphere

- Rt. anterior cerebral artery

6235.0 Rt. frontal cortex

7.6 Rt. frontal cortex

$3 \quad 35.0$ Rt. frontal cortex

22.0 Rt. frontal cortex

6.8 Rt. frontal cortex

13.2 Rt. frontal white matter

$12.7 \mathrm{Lt}$. frontal white matter

$7 \quad 33.4$ Rt. parietal cortex

12.8 Rt. parietal cortex

6.7 Rt. cerebellar hemisphere

7.2 Lt. cerebellar hemisphere

- Lt. middle cerebral artery

560 M Aphasia, right hemiparalysis

$<42117.0$ Lt. cerebral hemisphere

5.5 Rt. parietal cortex

7.6 Lt. occipital white matter

6.8 Lt. occipital cortex

$6 \quad 67 \quad$ M Dysarthria, left upper limb paralysis

$0.58 \quad 12.5$ Rt. cerebellar hemisphere

7.1 Rt. cerebellar hemisphere

6.1 Rt. cerebellar hemisphere

3.9 Rt. frontal white matter

4.6 Rt. frontal white matter

$7.4 \mathrm{Lt}$. frontal white matter

$\begin{array}{llll}7 & 79 & \text { F } & \text { Aphasia }\end{array}$

$144-150$

5.7 Lt. basal ganglia

$12.3 \mathrm{Rt}$. corona radiata

$4.2 \mathrm{Rt}$. corona radiata

$6.4 \mathrm{Lt}$. parietal cortex

$8 \quad 82 \quad M \quad$ Weakness of right upper limb

72-78 $3 \quad 2.9$ Lt. corona radiata

9.9 Lt. frontal white matter

8.0 Lt. parietal cortex

$19.3 \mathrm{Lt}$. thalamus

$9 \quad 91 \quad$ M Sudden collapse, dysarthria

$42-44$

$5 \quad 20.5$ Lt. hippocampal tail

17.0 Lt. occipital cortex

10.8 Lt. lateral white matter

$10 \quad 89 \quad F \quad$ Left hemiparesis

$110-120$

$11 \quad 86 \quad F \quad$ Weakness of left limbs

13$$
14
$$$$
15
$$$$
16
$$$$
1
$$

$17 \quad 78 \quad \mathrm{~F}$ Sudden weakness of right upper limb

$18 \quad 83 \quad \mathrm{~F}$ Unknown

Unknown

$\begin{array}{rr}1.5 & 2 \\ 9-10 & 1 \\ 2 & 2 \\ 5.5 & 1\end{array}$

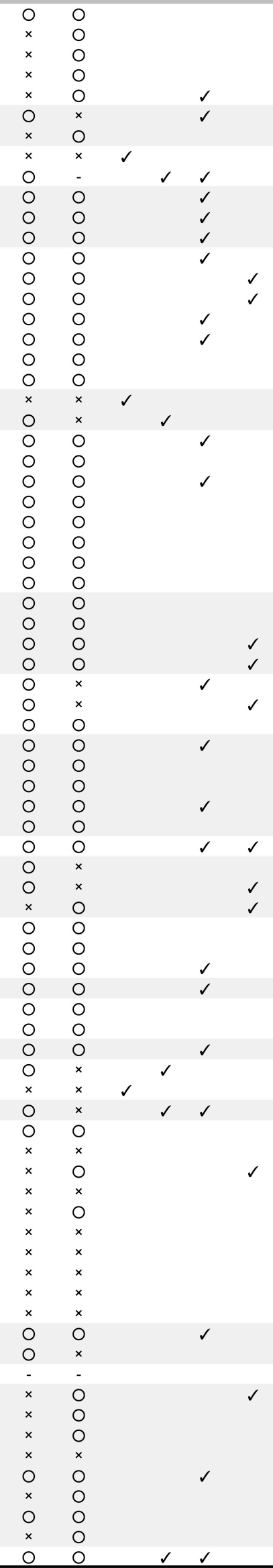

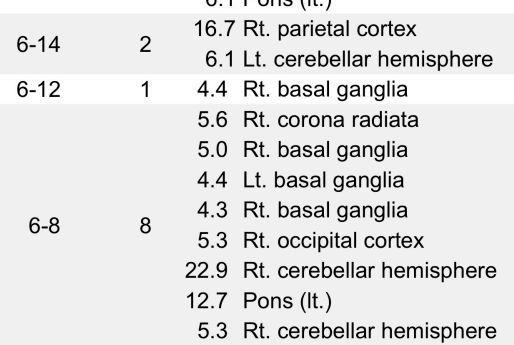

6.7 Rt. parietal cortex

4.4 Rt. basal ganglia

Rt. corona radiata

5.3 Rt. cerebellar hemisphere

34.2 Rt. frontal cortex
$7.9 \mathrm{Rt}$. insular white matter

7.6 Rt. frontal white matter

7.0 Rt. corona radiata

14.4 Rt. thalamus

18.4 Lt. hippocampus

16.4 Rt. occipital cortex

43.3 Lt. parietal cortex

30.6 Rt. frontal white matter

20.7 Lt. frontal white matter

28.6 Lt. insular cortex

48.1 Lt. cerebral hemisphere

- Lt. middle cerebral artery

19.9 Lt. lateral cortex

14.0 (rt.)

8.1 (It.)

3.8 Lt. corona radiata

4.1 Rt. thalamus

$6.2 \mathrm{Lt}$. thalamus

9.6 Lt. hippocampus

16.2 Pons (rt.)

18.0 Rt. cerebellar hemisphere

37.9 Lt. cerebellar hemisphere

6.1 Pons (It.) 
Total

Kyoto University ReseardMaleormation Repositony

Female

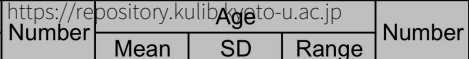

Number

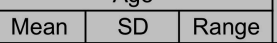

All positive patients

23

\begin{tabular}{l|c|c|c|}
\hline 77.5 & 16.7 & $11-92$ & 10 \\
\hline 74.7 & 19.6 & $11-91$ & 7
\end{tabular}

\begin{tabular}{|c|c|c|c|}
\hline 77.0 & 10.4 & $60-92$ & 13 \\
\hline 78 & 8.45 & $67-91$ & 7 \\
\hline
\end{tabular}

Mean 1 Age Age

Range

Mean

SD $\quad$ Range

DWI-positive only patients

14

\begin{tabular}{|l|l|l|}
74.7 & 19.6 & $11-91$ \\
\hline 79.3 & 8.93 & $49-91$
\end{tabular}

\begin{tabular}{l|l}
7 & 78 \\
\hline
\end{tabular}

\begin{tabular}{l|l}
8.45 & $67-91$ \\
\hline 5.07 & $73-87$ \\
\hline
\end{tabular}

77.8

20.7

11-92

300

443

1-1836

Negative patients

\begin{tabular}{l|l}
8.93 & $49-91$
\end{tabular}

\begin{tabular}{l|l}
10 & 79.8 \\
\hline
\end{tabular}

5.07

73-87

9

78.7

12.1

49-91

160

214 


\begin{tabular}{|c|c|c|c|c|c|c|c|c|c|c|c|c|c|c|c|c|c|c|c|}
\hline \multirow[b]{2}{*}{ Reader } & \multicolumn{2}{|c|}{ FOM } & \multicolumn{2}{|c|}{$\begin{array}{c}\text { Sensitivity } \\
\text { per lesion }\end{array}$} & \multicolumn{2}{|c|}{$\begin{array}{l}\text { Sensitivity } \\
\text { per case }\end{array}$} & \multicolumn{2}{|c|}{$\begin{array}{c}\text { Specificity } \\
\text { per case }\end{array}$} & \multicolumn{2}{|c|}{$\begin{array}{c}\text { False positives } \\
\text { per case }\end{array}$} & \multicolumn{2}{|c|}{$\begin{array}{c}\text { Reading Time } \\
(\mathrm{sec})\end{array}$} & \multicolumn{2}{|c|}{$\begin{array}{c}\text { Survey } \\
1 \\
\end{array}$} & \multirow{2}{*}{$\begin{array}{c}\text { Survey } \\
2 \\
\text { With } \\
\text { TS }\end{array}$} & \multicolumn{2}{|c|}{ FOM } & \multicolumn{2}{|c|}{$\begin{array}{l}\text { Sensitivity } \\
\text { per lesion }\end{array}$} \\
\hline & \begin{tabular}{|c|}
$\begin{array}{c}\text { Without } \\
\text { TS }\end{array}$ \\
\end{tabular} & $\begin{array}{c}\text { With } \\
\text { TS }\end{array}$ & $\begin{array}{c}\text { Without } \\
\text { TS }\end{array}$ & $\begin{array}{c}\text { With } \\
\text { TS }\end{array}$ & \begin{tabular}{|c|} 
Without \\
TS \\
\end{tabular} & \begin{tabular}{|c|} 
With \\
TS \\
\end{tabular} & \begin{tabular}{|c|} 
Without \\
TS \\
\end{tabular} & $\begin{array}{c}\text { With } \\
\text { TS }\end{array}$ & $\begin{array}{c}\text { Without } \\
\text { TS }\end{array}$ & $\begin{array}{c}\text { With } \\
\text { TS }\end{array}$ & \begin{tabular}{|c|} 
Without \\
TS
\end{tabular} & $\begin{array}{c}\text { With } \\
\text { TS }\end{array}$ & \begin{tabular}{|c|} 
Without \\
TS
\end{tabular} & $\begin{array}{c}\text { With } \\
\text { TS }\end{array}$ & & \begin{tabular}{|c|} 
Without \\
TS \\
\end{tabular} & $\begin{array}{c}\text { With } \\
\text { TS }\end{array}$ & \begin{tabular}{|c|} 
Without \\
TS
\end{tabular} & $\begin{array}{c}\text { With } \\
\text { TS }\end{array}$ \\
\hline 1 & 0.670 & 0.869 & 0.403 & 0.714 & 0.652 & 0.870 & 0.895 & 1.000 & 0.143 & 0.095 & 120 & 150 & 3 & 4 & 4 & \begin{tabular}{|l|}
0.429 \\
\end{tabular} & 0.738 & 0.241 & 0.368 \\
\hline 2 & 0.642 & 0.819 & 0.364 & 0.610 & 0.522 & 0.696 & 0.895 & 0.895 & 0.071 & 0.071 & 179 & 133 & 4 & 4 & 4 & 0.310 & 0.667 & 0.595 & 0.747 \\
\hline 3 & 0.617 & 0.795 & 0.338 & 0.688 & 0.565 & 0.913 & 1.000 & 0.947 & 0.357 & 0.214 & 173 & 197 & 2 & 3 & 4 & 0.381 & 0.738 & 0.298 & 0.711 \\
\hline 4 & 0.492 & 0.721 & 0.260 & 0.429 & 0.609 & 0.739 & 0.789 & 1.000 & 0.143 & 0.048 & 186 & 109 & 2.5 & 5 & 5 & 0.262 & 0.548 & 0.572 & 0.626 \\
\hline 5 & 0.576 & 0.793 & 0.208 & 0.610 & 0.478 & 0.783 & 1.000 & 0.895 & 0.071 & 0.095 & 124 & 138 & 3 & 3 & 4 & 0.190 & 0.571 & 0.639 & 0.833 \\
\hline 6 & 0.568 & 0.646 & 0.234 & 0.519 & 0.522 & 0.870 & 0.947 & 0.789 & 0.119 & 0.310 & 163 & 126 & 3 & 4 & 4 & 0.238 & 0.476 & 0.333 & 0.635 \\
\hline 7 & 0.348 & 0.645 & 0.195 & 0.416 & 0.522 & 0.739 & 0.474 & 1.000 & 0.762 & 0.048 & 301 & 249 & 3 & 3 & 3 & 0.167 & 0.429 & 0.491 & 0.786 \\
\hline 8 & 0.290 & 0.641 & 0.234 & 0.649 & 0.478 & 0.826 & 0.263 & 0.474 & 1.190 & 0.738 & 215 & 254 & 3 & 3 & 4 & 0.238 & 0.762 & 0.642 & 0.828 \\
\hline 9 & 0.583 & 0.862 & 0.325 & 0.727 & 0.609 & 0.826 & 0.895 & 1.000 & 0.167 & 0.024 & 223 & 221 & 3 & 3 & 3 & 0.357 & 0.786 & 0.635 & 0.878 \\
\hline 10 & 0.526 & 0.745 & 0.182 & 0.558 & 0.522 & 0.783 & 0.842 & 0.947 & 0.071 & 0.048 & 132 & 176 & 3 & 3 & 4 & 0.214 & 0.548 & 0.685 & 0.893 \\
\hline 11 & 0.623 & 0.724 & 0.208 & 0.468 & 0.522 & 0.783 & 1.000 & 0.947 & 0.048 & 0.119 & 119 & 121 & 3 & 3 & 4 & 0.190 & 0.500 & 0.513 & 0.754 \\
\hline 12 & 0.673 & 0.812 & 0.364 & 0.558 & 0.565 & 0.739 & 1.000 & 1.000 & 0.048 & 0.000 & 173 & 177 & 2 & 3 & 4 & 0.286 & 0.571 & 0.584 & 0.882 \\
\hline 13 & 0.210 & 0.400 & 0.117 & 0.325 & 0.304 & 0.522 & 0.947 & 0.842 & 0.024 & 0.143 & 138 & 129 & 2 & 2 & 4 & 0.167 & 0.286 & 0.565 & 0.784 \\
\hline 14 & 0.544 & 0.773 & 0.286 & 0.571 & 0.522 & 0.826 & 0.737 & 0.947 & 0.190 & 0.190 & 174 & 195 & 3.5 & 4 & 4 & 0.214 & 0.571 & 0.496 & 0.774 \\
\hline Overall & \begin{tabular}{|l}
0.526 \\
\end{tabular} & $0.732 *$ & 0.265 & $0.560^{*}$ & 0.528 & $0.780^{*}$ & 0.835 & 0.906 & 0.243 & 0.153 & 173 & 170 & 3 & 3 & 4 & 0.521 & $0.750^{*}$ & 0.260 & $0.585^{*}$ \\
\hline
\end{tabular}


Fig 1.

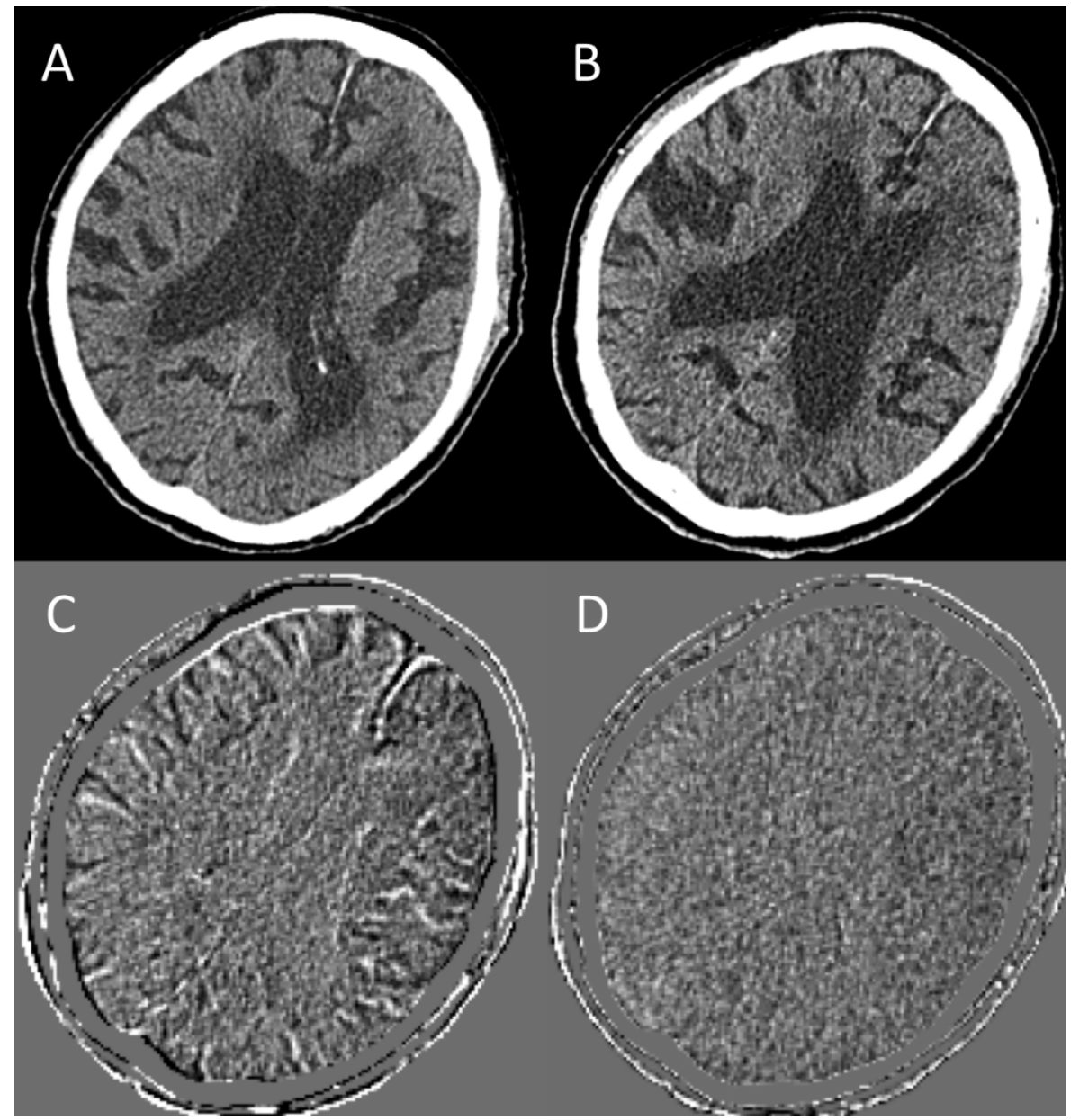


Fig 2.

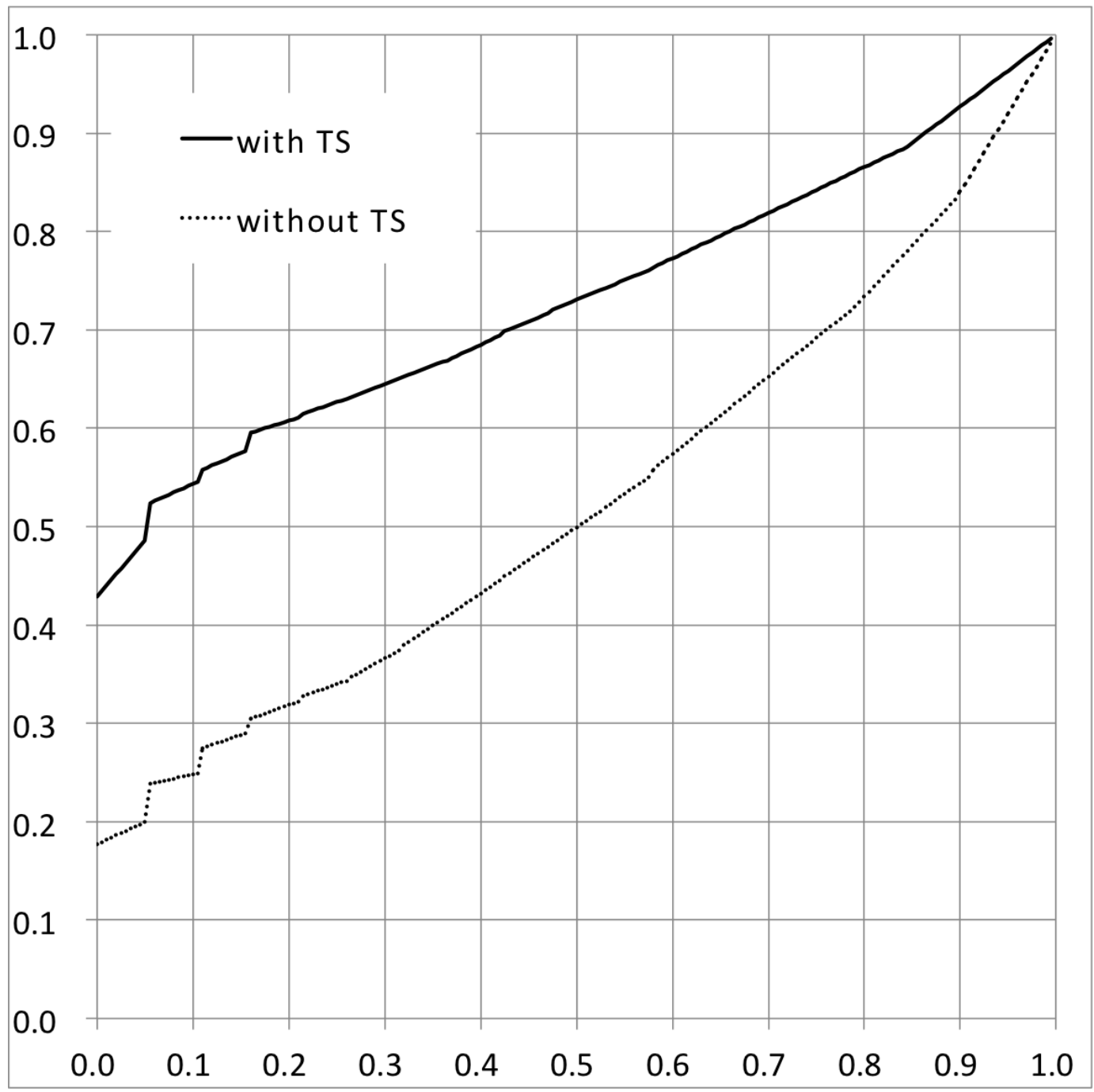


Fig 3.

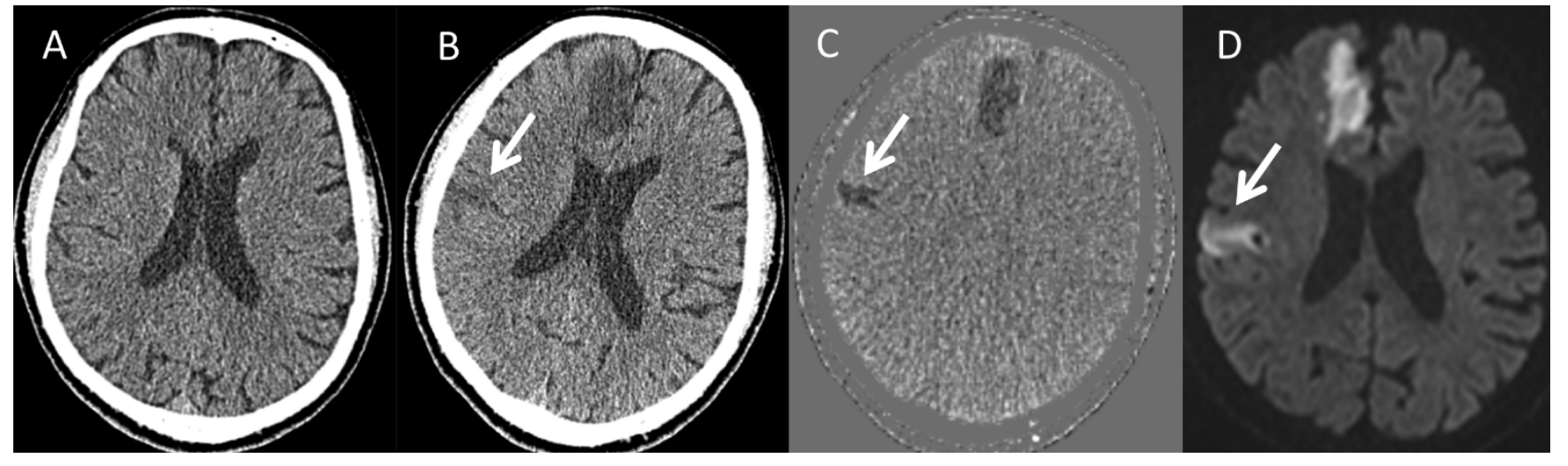


Fig 4 .

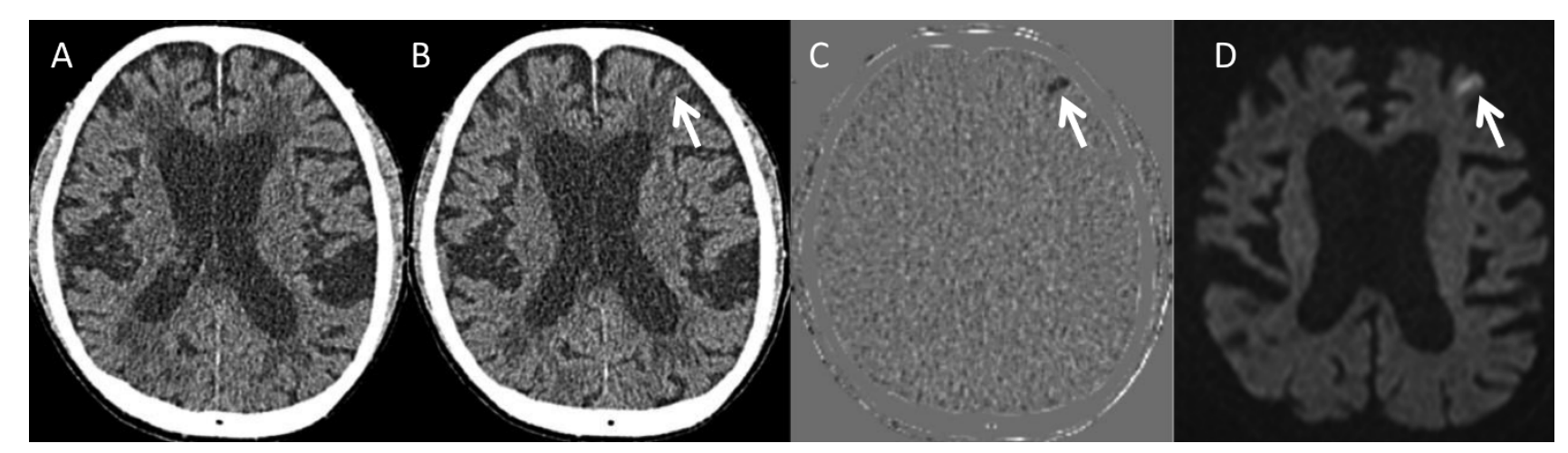


Fig 5.

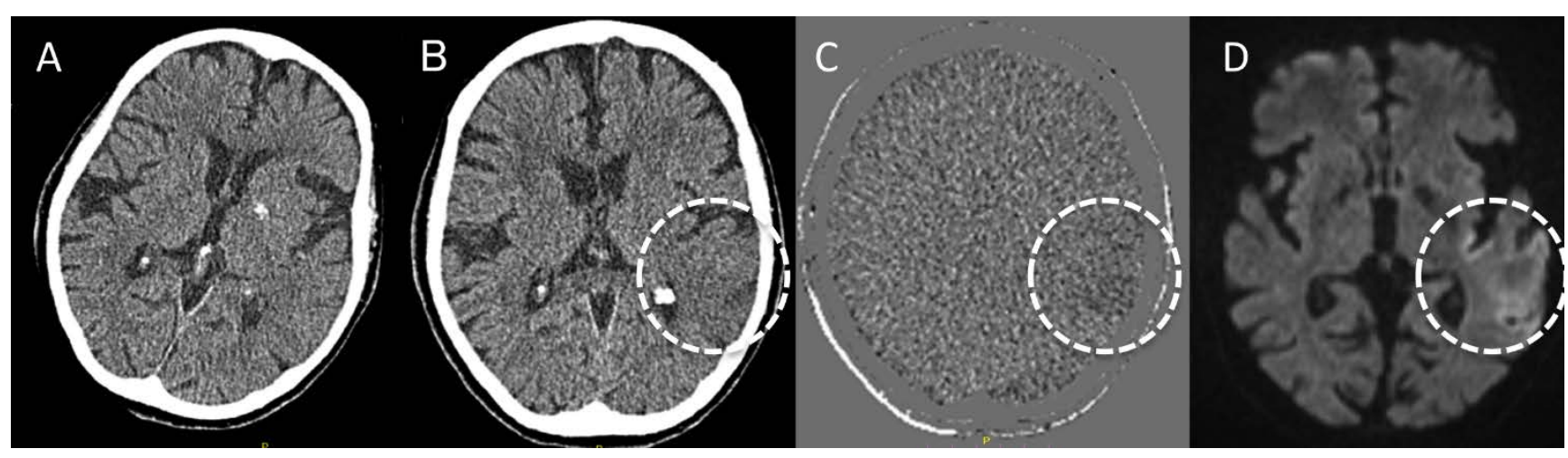


Fig 6.

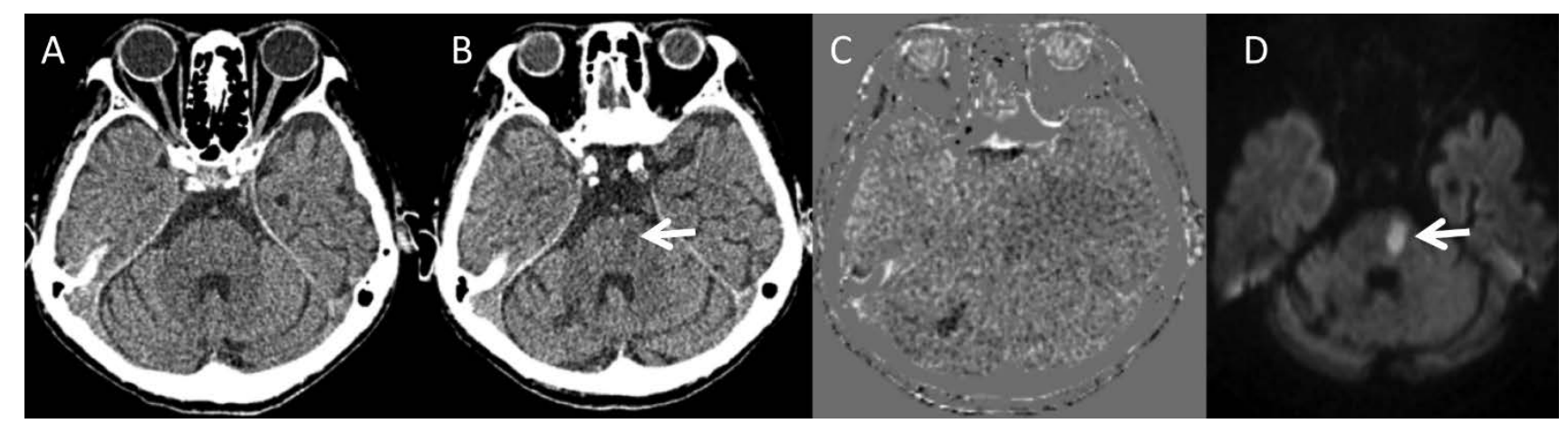




\section{SUPPLEMENTARY MATERIAL}

Detection of Brain Infarction on CT Can Be Significantly Improved With Temporal Subtraction Images

Thai Akasaka, Masahiro Yakami, Mizuho Nishio, Koji Onoue, Gakuto Aoyama, Keita

Nakagomi, Yoshio Iizuka, Takeshi Kubo, Yutaka Emoto, Kiyohide Satoh, Hiroyuki

Yamamoto, Kaori Togashi 


\section{Supplementary Methods}

\section{Temporal Subtraction Technique}

Temporal subtraction (TS) images were created fully automatically by subtracting the transformed previous images from the current images. Figure I shows a flowchart of the TS technique for the image transformation and subtraction. Here, all steps of image processing were done in the three-dimensional space. These steps are described in the following sections.

\section{Step 1: Head and Bone Area Segmentation}

The objective of this step was to extract the bone area from previous and current CT images. First, CT images from both time points were resampled to $1 \mathrm{~mm}$ isotropic voxels to reduce the data size and computational burden in the following image processes. Second, median filtering (mask size, $3 \times 3 \times 3 \mathrm{~mm}$ ) was performed to reduce noises on the CT images. Third, head area segmentation was performed based on a threshold ( -200 HU (Hounsfield unit)) with morphologic operations (opening and closing, radius $=1$ $\mathrm{mm}$ ) and a hole-filling process. The segmentation result of the head area was used as a processing mask in the following bone segmentation process. Moreover, in order to shorten the processing time in the following steps, the bounding box that fitted to the head area was automatically calculated for each previous and current CT image, and was used to crop the CT image. Fourth, a binary mask image that corresponded to the cortical bone area was extracted with a 250-HU threshold. Finally, the extracted bone area was refined with a morphologic closing process (radius $=20 \mathrm{~mm}$ ) to fill in holes (Figure II).

\section{Step 2: Rigid Registration}

Previous CT images were globally registered to current CT images via rigid transformation. The transformation matrix was calculated by using bone area segmentation results of the previous and current images from step 1 . The size of the previous CT images was matched with the size of the current CT images during this transformation process. 


\section{Step 3: Soft-tissue Emphasis}

A soft-tissue window setting (window level, $35 \mathrm{HU}$; window width, $150 \mathrm{HU}$ ) was applied to each resampled CT image. This process converted the value range of the CT images to 0-255, and emphasized the change in CT values of the soft-tissue region. By emphasizing soft-tissue region, our next step was able to capture more specific deformation needed to register brain structures of the previous and current CT images.

\section{Step 4: LDDMM Registration}

The images from step 3 were further registered by using the large deformation diffeomorphic metric mapping (LDDMM) algorithm [1, 2]. The LDDMM algorithm is one of the non-rigid image registration algorithms that originated from Pattern Theory [3], which was specifically designed for a large amount of deformation. It is used to compute a diffeomorphic transformation between two images while preserving the topology of the object. Thus, it has been studied over the past decades for use in applications in the area known as computational anatomy [4]. Past studies have shown its strength in magnetic resonance imaging neuroscience and biomedical engineering [5-7].

To deal with large displacement between the previous and current CT images, LDDMM was performed by using cascading processing, in which the elasticity of transformation was gradually increased [5, 6]. In addition, the cascading processing was performed in a coarse-to-fine fashion to reduce computational loads. All of the parameters of LDDMM were empirically determined, and the same parameters were used for all input image pairs in our experiment.

\section{Step 5: Subtraction with Voxel Matching Method}

TS images were obtained by subtracting the transformed previous images from the current images. Note that this process was performed by using soft-tissue-emphasized images. Thus, the subtraction was not measured in Hounsfield unit. In addition, for the reduction of subtraction artifacts, the voxel-matching technique [8] was employed during 
the calculation of subtraction. The kernel size for the voxel-matching process was set as 0.3 to $0.8 \mathrm{~mm}$ depending on the resolution of the input pair of CT images.

\section{CT Images and Application of Temporal Subtraction Technique}

All CT images were acquired with multidetector CT scanners (Aquilion 64, Aquilion PRIME, Aquilion ONE; Toshiba Medical Systems, Otawara, Japan). A head kernel (FC67) was used as the reconstruction kernel for all 84 CT examinations (42 pairs). The size of the CT images was $512 \times 512$ pixels and tube voltage was $120 \mathrm{kVp}$. Tube current were $300 \mathrm{~mA}$ for $80 \mathrm{CT}$ examinations, $400 \mathrm{~mA}$ for two examinations, $270 \mathrm{~mA}$ for one examination, and $65 \mathrm{~mA}$ for the remaining one examination. Gantry rotation time was 750 msec for 83 CT examinations and $1000 \mathrm{msec}$ for the remaining one examination. CT images were reconstructed with a $210 \mathrm{~mm}$ field of view (FOV) for 82 CT examinations, and FOVs of $198.75 \mathrm{~mm}$ and $209.375 \mathrm{~mm}$ were used for the remaining two CT examinations. The slice thickness ranged from 0.3 to $0.8 \mathrm{~mm}$ (mode $=0.3 \mathrm{~mm}$ ), and 7 out of 42 image pairs had different slice thickness for the previous and current CT images.

Average processing time was 21.5 minutes for the whole calculation process of TS images of one pair of CT images. All the steps of image processing were conducted on a desktop computer (Intel Core i7-6700K central processing unit 4.00 GHz, 32.0 GB memory; Intel, Santa Clara, California). Parallelized computing was implemented, and the amount of memory used was approximately $3 \mathrm{~GB}$, depending on the size of the image. These steps of image processing were implemented as a pipeline by Canon Inc., and it is a proprietary software designed for our study. 
Supplementary Figure I. Flowchart of our temporal subtraction method.

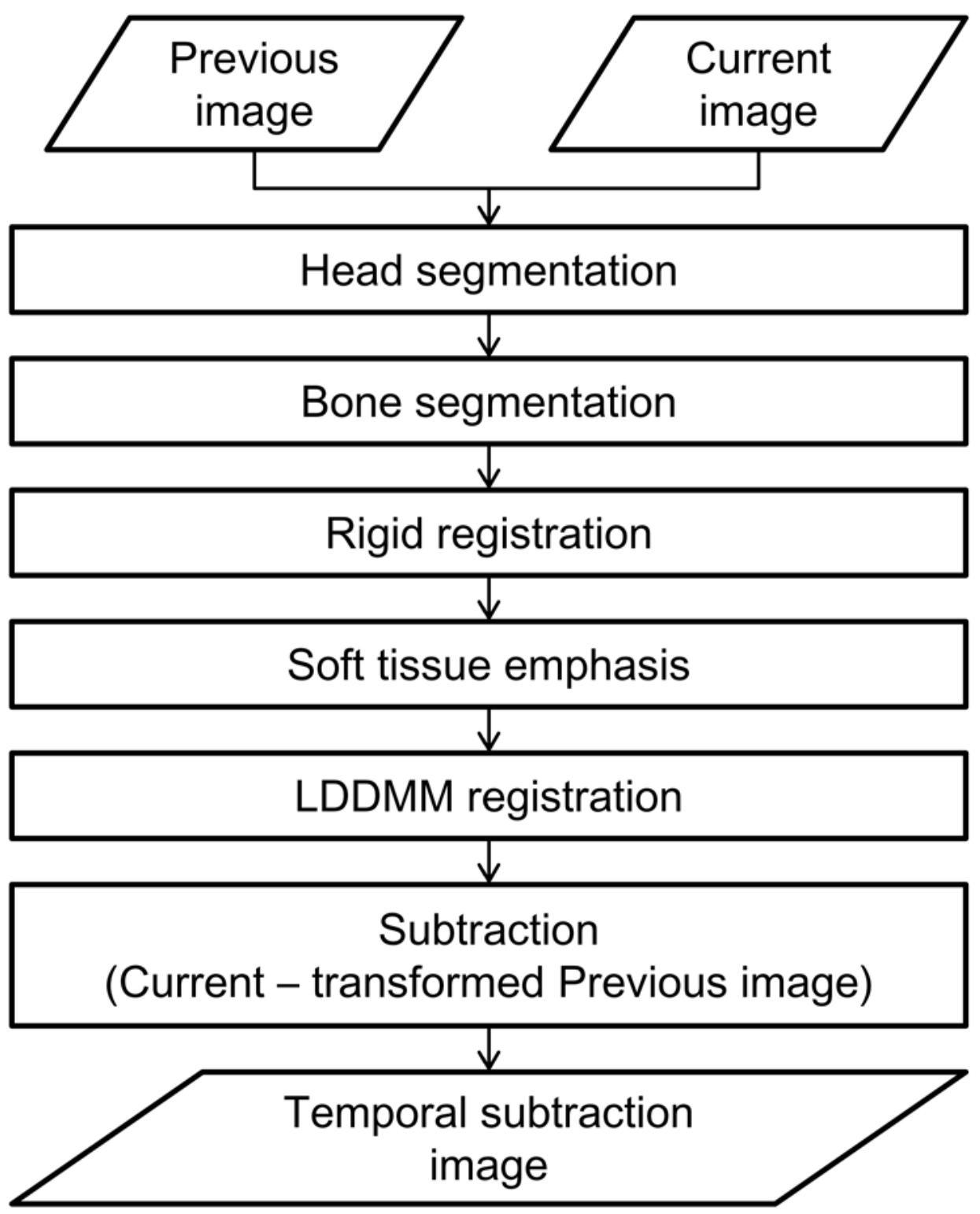


Supplementary Figure II. Examples of the image processing results of our temporal subtraction method. A, CT image after resampling ( $\mathrm{WL}=35, \mathrm{WW}=250), \mathrm{B}$, extracted bone area, and C, soft tissue-emphasized image ( $\mathrm{WL}=127$, WW $=256)$.

(A)

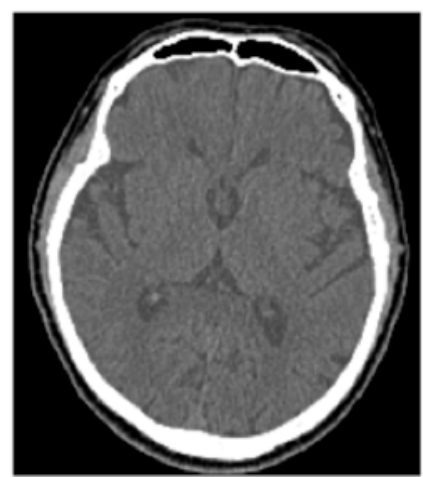

(B)

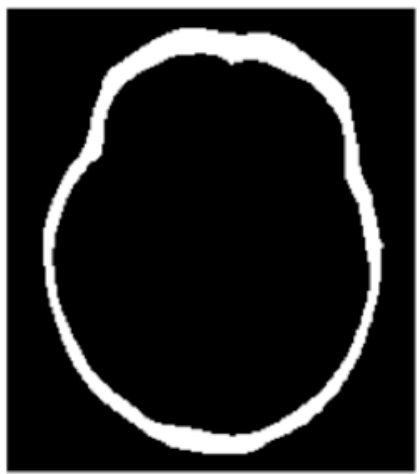

(C)

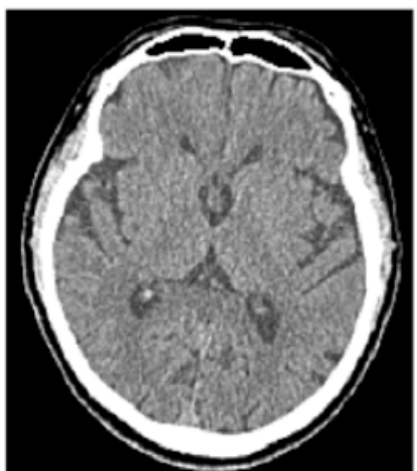




\section{Supplementary References}

1. Beg MF, Miller MI, Trouvé A, Younes L (2005) Computing Large Deformation Metric Mappings via Geodesic Flows of Diffeomorphisms. Int J Comput Vis 61:139-157 . doi: 10.1023/B:VISI.0000043755.93987.aa

2. Software at CIS : lddmm-volume: Namespace. http://cis.jhu.edu/software/lddmm-volume/namespace.php. Accessed 4 Nov 2016

3. Grenander U, Miller MI (2007) Pattern theory: From representation to inference. Oxford University Press

4. Miller MI, Qiu A (2009) The emerging discipline of Computational Functional Anatomy. Neuroimage 45

5. Sakamoto R, Mori S, Miller MI, et al (2014) Detection of time-varying structures by Large Deformation Diffeomorphic Metric Mapping to aid reading of high-resolution CT images of the lung. PLoS One 9: . doi: 10.1371/journal.pone.0085580

6. Ceritoglu C, Tang X, Chow M, et al (2013) Computational analysis of LDDMM for brain mapping. Front Neurosci. doi:

10.3389/fnins.2013.00151

7. Ceritoglu C, Oishi K, Li X, et al (2009) Multi-contrast large deformation diffeomorphic metric mapping for diffusion tensor imaging. Neuroimage 47:618-627 . doi: 10.1016/j.neuroimage.2009.04.057

8. Itai Y, Kim H, Ishikawa S, et al (2010) Development of a voxel-matching technique for substantial reduction of subtraction artifacts in temporal subtraction images obtained from thoracic MDCT. J Digit Imaging 23:31-38 . doi: 10.1007/s10278-008-9169-1 